\title{
A Full Time Domain Methodology based on Near Field Time Reversal for Equivalent Source Identification
}

\author{
Sassia Hedia ${ }^{(1),(2)}$, Bessem Zitouna ${ }^{(1)}$, Jaleleddine Ben Hadj Slama ${ }^{(1)}$, and Lionel Pichon ${ }^{(2)}$ \\ ${ }^{(1)}$ Laboratory of Advanced Technology and Intelligent Systems, National Engineering School, \\ University of Sousse, Tunisia. \\ ${ }^{(2)}$ Group of Electrical Engineering, Paris (GeePs), CNRS UMR 8507, Centrale Supélec, \\ University of Paris-Sud, University of Paris-Saclay, France. \\ E-mail : sassia.hedia @u-psud.fr
}

\begin{abstract}
This paper deals with magnetic source characterization in time domain. The basic idea is to solve the inverse problem using the measured near field radiation cartography. In order to ensure the identification procedure, the Time Reversal (TR) technique is used. This procedure allows both the spatial and temporal focusing determination by forcing waves to virtually converge to their initial source. The originality of the proposed methodology is to present a full time domain study of a magnetic source reconstruction. Indeed, this approach is particularly suitable for structures that emit non-sinusoidal radiations such as power electronic systems. First, the Electromagnetic Time Reversal (EMTR) basis are introduced. Then a simulation case study is discussed. Finally, results from an experiment test are presented to verify the proposed methodology. The measured results are in good agreement with the calculated electromagnetic fields. The experimental validation shows that compared to other identification techniques, especially those developed in the frequency domain, the proposed approach is more efficient and simple.
\end{abstract}

Keywords - Electromagnetic radiation, Inverse problem, Near field, Time Domain, Time Reversal technique.

\section{INTRODUCTION}

The significant increase in the clock frequency of embedded electronic systems, has led to serious problems of electromagnetic interference between components. Hence, the consideration of Electromagnetic Compatibility (EMC) modelling techniques has become a crucial solution to deal with the unwanted interferences and improve systems design. In electronics industry, devices are especially affected by noisy transient signals [1]. Therefore, to cope with the harmful Electromagnetic (EM) coupling, researchers have developed many characterization approaches for emissive systems.

To carry out an efficient EMC study, it is useful to know the EM field distribution in a near region around the circuit under test. Actually, the near field technique is considered as an appropriate approach to characterize radiating structures [1]-[9]. But, due to the expensive measurements cost, this task relies on the use of a robust numerical process. In fact, the need to avoid heavy simulations realization has pushed researchers to develop new behavioural models [1]-[6]. Recently, the EM inverse problem has shown interest in characterizing radiated sources [1]-[9]. Generally, this method is used to obtain the current flowing through emissive sources using a set of EM elemental dipoles. In [2], authors have suggested the coupling of the EM inverse problem based on Genetic Algorithms (GA) with the Moment method in the Frequency Domain (FD). The proposed hybrid process has the advantage to identify rectangular structures. In [3], authors applied the Artificial Network and the Pseudo Zernike Moment Invariant and presented a parametric study to evaluate the influence of the different parameters such as measurement resolution and height. This methodology was improved, in [4], by use of GA in the FD in order to accelerate the convergence of the algorithm. To enhance the computational time, Benyoubi et al. [5], proposed a fast technique based on the matrix inversion and an optimization procedure, which allows finding equivalent sources with a smaller number of dipoles, compared to [6]. In [7], FD EM inverse problem is elaborated to evaluate the shielding effectiveness of perforated plates. However, to determine properties of structures that work in the transient regime, it is more appropriate to characterize the system in the Time Domain (TD) rather than the FD. Indeed, in transient signals, radiation is concentrated within a short period while it is spread in a wider frequency spectrum. Hence, characterizing wideband structures is both efficient and easier to in the TD.

In the literature, works using inverse problem resolution with a TD analysis begin to be developed. Among the studies recently published on this topic, the research presented in [8] and improved in [9], presents the theoretical foundation of TD EM inverse method and uses the GA as an optimization solution. In this study, the identification procedure is carried out by extracting at each iteration a local cartography, starting from the highest magnetic field until the weakest radiation. Unlike works devoted in the FD and that treat sinusoidal waveforms, authors in [10] dealt with structures that emit non-sinusoidal signals in the TD. Such methodology is conclusive, but it is a 
time consuming task. There are other methods for TD characterization, such as the stochastic approach studied in [11]. Generally, works based on TD analysis relies on the time recording of the data using a high-resolution oscilloscope. But, this information is usually treated using a spectral study [11].

In this work, we propose to reconstruct the whole radiation propagating process in TD in order to follow signal changes over time and detect the disturbances contribution. The objective of this paper is to develop a simple and a low cost EM characterization method based on the use of analytical expressions of equivalent dipoles in TD. The basic idea of the proposed approach is to introduce the Time Reversal (TR) technique in order to force EM waveforms to converge to their original sources. Due to its focusing properties, the TR process is widely used on acoustic communications, its pristine application [12]. Then, this technique was successfully applied in electromagnetism [13]. It is used for buried objects localization [14], for reverberation chamber applications [15], for faults location [16], and for microwave imaging [18]. In the literature, TR studies rely on the use of the Decomposition of the TR Operator (DORT) method for location issues through the TR Operator eigen vectors analysis [12]. Nevertheless, the standard DORT has shown a weakness in providing selective focusing on each of the emissive sources separately within a circuit where sources are radiating at the same time [17]. In order to overcome the drawbacks of the DORT technique, research works moved toward a more suitable approach, which is the TD analysis. Working in TD has the advantage of giving the data behaviour over a period. The basic thought behind the applicability of the TR technique in the TD is the determination of the instant of focus at which the most suitable field distribution is obtained [14]. Indeed, in the most of TR studies based on TD analysis, the measured signals are realized in the FD, then numerically transformed to the TD [19]. However, Kosmas et al. [18], developed a full TD method based on the TR algorithm for breast cancer detection. The Finite Difference TD has been applied to achieve the backpropagation operation and make waves converge back to the effective source. Likewise, in [13], authors have used the TR in Transmission Lines network for the identification of defects in cables and for lighting location. By analogy to this work, the present study is completely performed in TD, which is a relatively unexplored area in EMC. In this work, we propose to use the Electromagnetic Time Reversal (EMTR) technique to localize and identify disturbances source in an electronic system. Actually, the measured radiated EM field contains several of information that could be extracted using the TR technique. The aim of the proposed methodology is to determine the equivalent dipole parameters, such as excitation signal and geometry.

This paper introduces a simple approach for EM emissions representation using the equivalent radiation expressions with the near field scanning technique. The proposed TD inverse method is based on the EMTR technique. Section II is dedicated to analytical investigations. In particular, both EM inverse problem and EMTR are studied. In section III, the proposed methodology is applied on a simulation case study. In section IV, the experimental test bench is presented in order to validate the proposed approach.

\section{ANALYTICAL INVESTIGATIONS}

\section{A. Electromagnetic Inverse Problem}

It is generally useful to provide efficient equivalent models to obtain radiated emissions without reference to complicated design or use of commercial solvers. The EM inverse problem permits fields simulation, in order to identify structures parameters that give the same magnetic field $H$, such as position, and excitation form. By analogy to the FD, analytical expressions of equivalent dipole radiation were developed in TD [8]. The magnetic source, of radius $r$, with center coordinates $\left(X_{d}, Y_{d}, Z_{d}\right)$ and angles of orientations $\left(\theta_{d}, \varphi_{d}\right)$, is presented in the near field region in Fig.1.

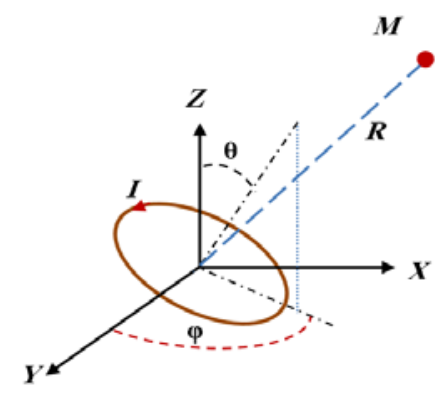

Fig. 1. Equivalent magnetic dipole definition.

In this work, magnetic field components $H_{x}, H_{y}$ and $H_{z}$ are calculated in a simplified way, in the cartesian coordinates system, at measurement point $\left(X_{0}, Y_{0}, Z_{0}\right)$, as follows:

$$
H_{x, y, z}=A \times\left[\left(B \times C_{x, y, z}\right)-\left(D \times E_{x, y, z} \times F\right)\right]
$$

where:

- $A=\frac{r^{2}}{4 R} \cdot I_{0}$

- $B=\left(\left(\frac{1}{c^{2}} \cdot \frac{\partial^{2} I\left(t^{\prime}\right)}{\partial t^{2}}\right)+\left(\frac{1}{c R} \cdot \frac{\partial I\left(t^{\prime}\right)}{\partial t}\right)+\left(\frac{1}{R^{2}} \cdot I\left(t^{\prime}\right)\right)\right)$

- $\quad C=\left(C_{x}, C_{y}, C_{z}\right)$

$$
=(\sin (\theta) \cos (\varphi), \sin (\theta) \sin (\varphi), \cos \theta)
$$

- $D=\left(\left(\frac{1}{c^{2}} \cdot \frac{\partial^{2} I\left(t^{\prime}\right)}{\partial t^{2}}\right)+\left(\frac{3}{c R} \cdot \frac{\partial I\left(t^{\prime}\right)}{\partial t}\right)+\left(\frac{3}{R^{2}} \cdot I\left(t^{\prime}\right)\right)\right)$

- $E=\frac{1}{R^{2}} \times\left(E_{x}, E_{y}, E_{z}\right)$

$$
=\frac{1}{R^{2}} \times\left(\left(X_{d}-X_{0}\right),\left(Y_{d}-Y_{0}\right),\left(Z_{d}-Z_{0}\right)\right)
$$

- $F=\left(\begin{array}{c}\cos (\theta)\left(Z_{d}-Z_{0}\right)+ \\ \sin (\theta) \sin (\varphi)\left(Y_{d}-Y_{0}\right)+ \\ \sin (\theta) \cos (\varphi)\left(X_{d}-X_{0}\right)\end{array}\right)$

and:

- $t^{\prime}=t-\frac{R}{c}$ is the delay time variable.

- $R$ is the distance between the measurement point and the center of the elementary dipole:

$$
R=\sqrt{\left(X_{d}-X_{0}\right)^{2}+\left(Y_{d}-Y_{0}\right)^{2}+\left(Z_{d}-Z_{0}\right)^{2}}
$$

- $I(t)$ is the instantaneous current signal with maximum magnitude $I_{0}$. 


\section{B. Electromagnetic Time Reversal}

The final purpose of the present paper is to take advantage of the TR procedure in predicting emissive elements positions. By analogy to the acoustics demonstration, this process was successfully applied to EM transients [13] [19]. Assume a magnetic source $\left(R_{0}\right)$ emits a pulse, with a period $\Delta \mathrm{t}$, propagates in the environment then measured and recorded using a transducers network $\left(R_{i}\right)$, as receivers, $1 \leq i \leq M$ with $M$ is the total sensors number. This array is called the Time Reversal Mirror (TRM). Afterwards, the recorded signal, at each measurement point, is totally flipped over in time then retransmitted through each transducer, as emitters, which is known as the back propagation process. Applying the TR procedure allows to maximize the received signal at a certain instant of time and to focus the field in space at rightly the original source location $\left(R_{0}\right)$ [12-15]. In TD, the main operation corresponds to the inversion of the sign of time, which is the following transformation:

$$
t \rightarrow-t
$$

In fact, the recorded data follows a Last-In, First-Out stack. Therefore, the returned wave propagates in the medium on its turn and acts as if it replays accurately its same past life. Based on the principle of the reciprocity, the TR experience requires a reversible propagation medium, in a way that if a field is propagated in this environment then its time reversed version must react just the same [12-15]. In other words, if $\phi(t)$ is a solution of the propagation equation, then $\phi(-t)$ is also a solution. So that the whole scene can be played from time $t=0$ or even replayed in reverse from the time $t=\Delta \mathrm{t}, t \in[0, \Delta \mathrm{t}]$. Consequently, the reversed signals will be recorded during an experiment time $\Delta \mathrm{t}$, then returned and entirely reemitted in a reversed chronology during $(\Delta t-t)$. The magnetic field received, at the instant $t$ and the position $p_{0}$, is defined by $H\left(p_{0}, t\right)$. Its reverse version is $H\left(p_{0}, \Delta t-t\right)$. According to [13] and [15], if we consider $T_{T R}$ the time inversion operator, we have the following transformation:

$$
T_{T R}\left\{H\left(p_{0}, t\right)\right\}=-H\left(p_{0},-t\right)
$$

In the near field region, the radiated signal received by a TRM transducers can be written as:

$$
H_{x, y, z}(t)=k\left(t, R_{0} \rightarrow R_{i}\right) \otimes I(t)
$$

where $\otimes$ is the convolution product, $k\left(t, R_{0} \rightarrow R_{i}\right)$ is the impulse response of the propagation medium at $R_{i}$ for a pulse emitted from $R_{0}$ and $I(t)$ is the excitation form. Indeed, this propagation matrix is constructed analytically, using a virtual array of potential sources placed on the studied structure and excited by a delta-function signal. The reemission of the reversed received field from $R_{i}$ allows focusing signals at $R_{0}$. The temporal focused signal at $R_{0}$ takes the following form:

$$
E_{T R}\left(R_{0}, t\right)=\sum_{i=1}^{M} k\left(R_{i} \rightarrow R_{0}, t\right) \otimes H_{x, y, z_{i}}(-t)
$$

Evaluating the focusing quality is quite interesting. Actually, at the focusing time, the original source is illuminated and the radiated signal reaches its maximum peak. In the literature, several criteria are proposed to discuss the temporal and spatial focusing. In this paper, the maximum magnitude of the focused signal is used. In practice, we consider the absolute value of this useful part of the reconstructed wave, as follows:

$$
\operatorname{Max}\left(R_{0}\right)=\max _{t \in T}\left(\left|E_{T R}\left(R_{0}, t\right)\right|\right)
$$

The shape of the original current $I(t)$ corresponds to the reserved focused signal $E_{T R}\left(R_{0},-t\right)$ at the source location $R_{0}$.

\section{VALIDATION USING CALCULATED CARTOGRAPHIES}

In this section, a simulation test case is presented to validate the proposed approach. In addition, calculated cartographies help eliminating practical problems such as those related to the experimental measurements errors in the near field region. The studied structure is a surface of $(200 \mathrm{~mm} \times 200 \mathrm{~mm})$. Magnetic field calculations were performed at a height of $4 \mathrm{~cm}$ above the system under test. These calculation points constitute the TRM, with a displacement step of $4 \mathrm{~mm}$. This network is composed of 2601 transducers uniformly distributed on a $(X Y)$ plan. The radiating source is a current loop of a radius $25 \mathrm{~mm}$, located at the position $(0,0,0)$, Fig. 2 .

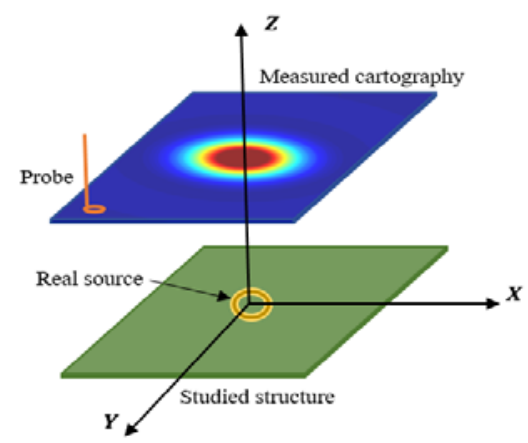

Fig. 2. System setup.

Since the principal idea behind the use of the TD method rather than the FD is to deal with non-sinusoidal signals in emissive structures and validate our approach. The dipole is crossed by a Gaussian type current, of a $10 \mu s$ duration and a $10 \mathrm{MHz}$ frequency (Fig.3a). Its expression is given by Eq.12:

$$
\mathrm{I}(t)=I_{0} \frac{\left(\mathrm{t}_{0}-\mathrm{t}\right)}{\tau} \exp \left(\frac{-\left(\mathrm{t}-\mathrm{t}_{0}\right)^{2}}{(2 \tau)^{2}}\right)
$$

where $\tau=7 \mu \mathrm{s}, \mathrm{t}_{0}=5 \tau$, and $\mathrm{I}_{0}=1 \mathrm{~A}$.

To construct the impulse response of the system, a network, with a resolution of $9.5 \mathrm{~mm}$, is placed on the studied structure. This network is of 441 equivalent sources that potentially could refer to the real source behavior. According to our mesh, the loop corresponds to the source number 221, which has the coordinates $(0,0,0)$. In fact, position control is used to validate the approach. The transient magnetic field is calculated using Eq.1. Actually, radiated signals are obtained at the TRM level in order to assimilate to measurements. Fig.3b shows the temporal radiated signals at different positions. The radiated field cartographies are obtained at each instant of the experiment, which describe the radiation propagation film. The magnetic components distributions are given in Fig.4. From Fig.5.a, we notice that the calculated field at point 221 , which corresponds to the real source position, has the highest magnitude. Thus, the effective dipole coordinates are correctly found. 
By applying the TR technique based on the maximum magnitude criteria, Fig.5a, the proposed approach permits to reconstruct the original excitation current signal presented in Fig.5b. The reconstructed $H_{z}$ signals, using the obtained excitation, shows a good agreement with the calculated field, at different positions, Fig.5c. In addition, calculated and estimated field cartographies, presented in Fig.4 and Fig.6, seem close one to another at each instant of the experiment.

a)

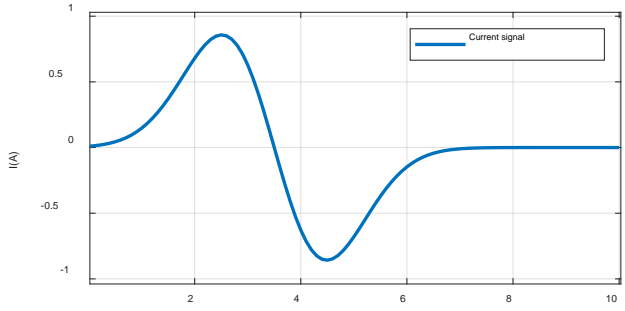

$\mathrm{t}(\mathrm{s})$

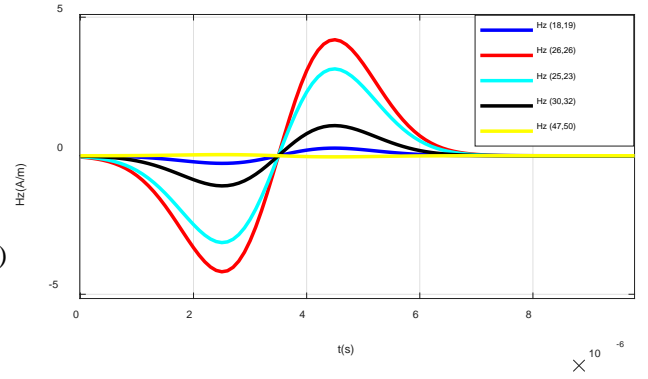

Fig. 3. (a) Excitation current $I(t)$, (b) Calculated $H_{z}$ at different points

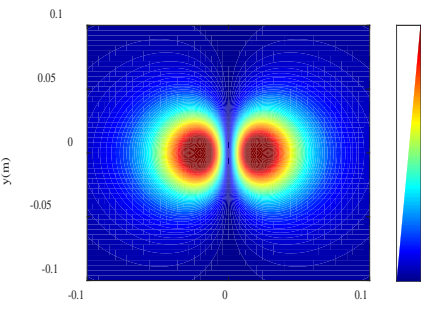

$x(m)$

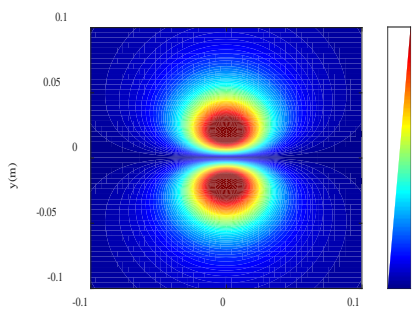

b) $H_{y}$ a) $H_{x}$

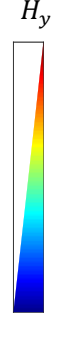

$\mathrm{x}(\mathrm{m})$

c) $H_{Z}$

Fig. 4. Calculated magnetic field distributions at $t=4,64 u \mathrm{~s}$.

c)

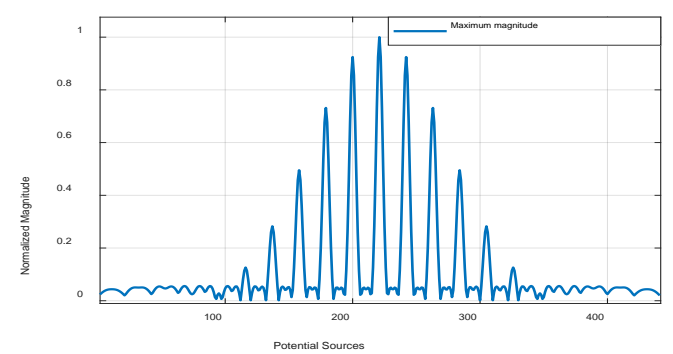

a)

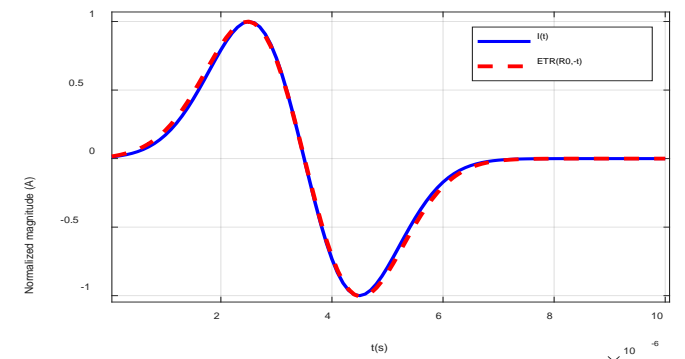

b)

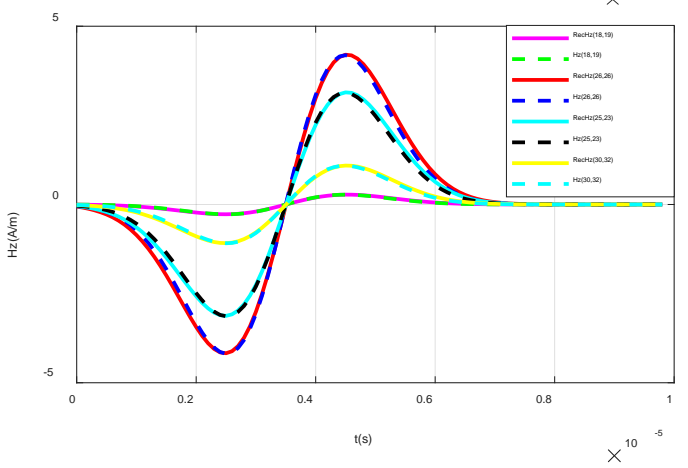

Fig. 5. (a) Focusing magnitudes, (b) Current, (c) Reconstructed fields

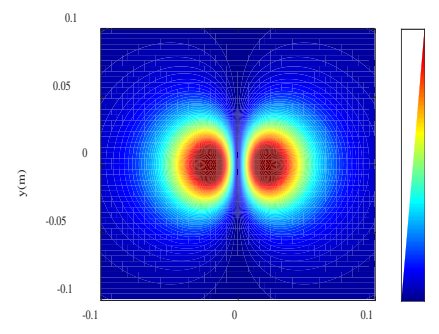

$\mathrm{x}(\mathrm{m})$

a) Reconstructed $H_{x}$

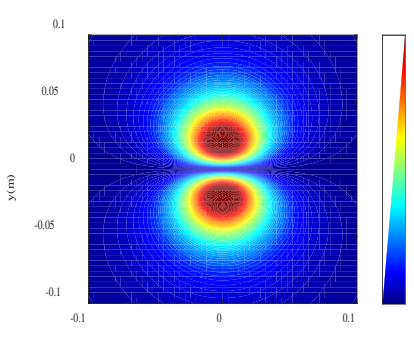

$x(m)$

b) Reconstructed $H_{y}$

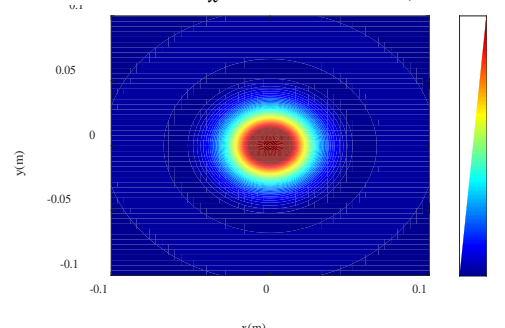

c) Reconstructed $H_{z}$

Fig. 6. Reconstructed field distributions at $t=4,64 u s$.

\section{VALIDATION USING MEASURED CARTOGRAPHIES}

In order to test the efficiency of the proposed approach, we suggest validating the TD EMTR method by an experimental measurement process above an elementary structure. The reason behind this choice is that we want to emulate the basic behaviour of a power system by the mean of a magnetic dipole carrying a non-sinusoidal current.

\section{A. Time Domain Near-Field test bench}

The measurement of the magnetic emissions in the TD using a near field scanning technique usually requires a high precision oscilloscope that displays and records the measured fields. Signals are captured by a magnetic field probe that moves above the system under test. The measurement were performed on a mono turn coil that was printed on a circuit 
board of surface $(21 \mathrm{~mm} \times 21 \mathrm{~mm})$, Fig.7. The loop radius is $5 \mathrm{~mm}$. The excitation signal is the current out coming from a chopper circuit, which provides a high current and leads to severe EM interference issues that badly affect the nearby power devices. In point of fact, this current signal has a nonsinusoidal waveform. Therefore, its modelling using the frequency method is not so evident. This is a real case where the use of the temporal method is strongly required. The magnetic probe is at a height of $8.5 \mathrm{~mm}$ and moves with a $1.5 \mathrm{~mm}$ resolution, Fig.7. The measurement network, 225 points, constitute the TRM. Indeed, the radiation quantity measured during the experimental period is the sum of the contributions captured from each transducer.

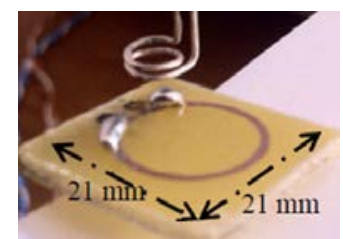

Fig. 7. Measuring $H_{z}$ magnetic field antenna, placed above the studied circuit.

\section{B. Results}

The transient measured signals, at the probe terminal, during the switching of the transistor are presented in Fig. 8. The magnetic fields at the Turn On of the transistor are presented in Fig.11.b. The corresponding radiated field cartography of the component $H_{z}$ is displayed in Fig.10.a. The application of the proposed method shows that the potential source, which has the maximum magnitude, Fig.9.a, is a real radiating source. In Fig.9.b, the obtained current signal is presented.

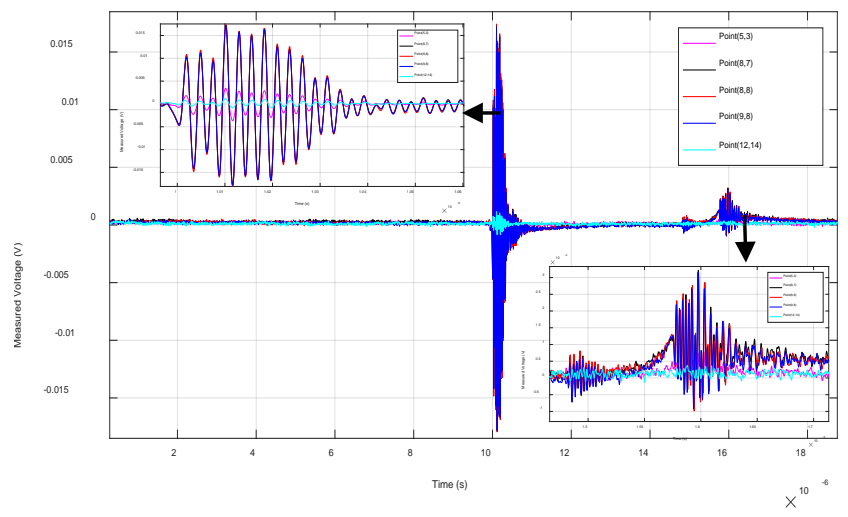

Fig. 8. Measured Voltage at the terminal of the probe

Based on the identified source coordinates, we observe in Fig.11.a. a good correlation between the reconstructed shape and the measured $H_{z}$ at the center position, at the Turn On of the chopper transistor. However, as a matter of fact, there are some lag time between the too signals and differences in the magnitude, that may be explained by the measurement possible errors, due to the coupling between the magnetic probe and the current loop. With using the obtained current signal, Fig.9.b, which has a non-sinusoidal form, we calculate the magnetic field signals at different position in Fig.11.c. Actually, the measured signals, in Fig.11.b, are close to the reconstructed waveforms presented in Fig.11.c at the same positions. Also, the reconstructed magnetic field cartography, Fig.10.b, is in good agreement with the measured distribution, Fig.10.a, at $\mathrm{t}=$ $10.3 \mu \mathrm{s}$. In fact, the proposed methodology provides the electromagnetic radiation at each instant of time throughout the experiment. Indeed, the main advantage of using the TD analysis is measuring transient events and disturbances that appear when switching ON or OFF, which is not evident when employing frequency instrumentation. In addition, the source position and its corresponding current signal are obtained.

a)

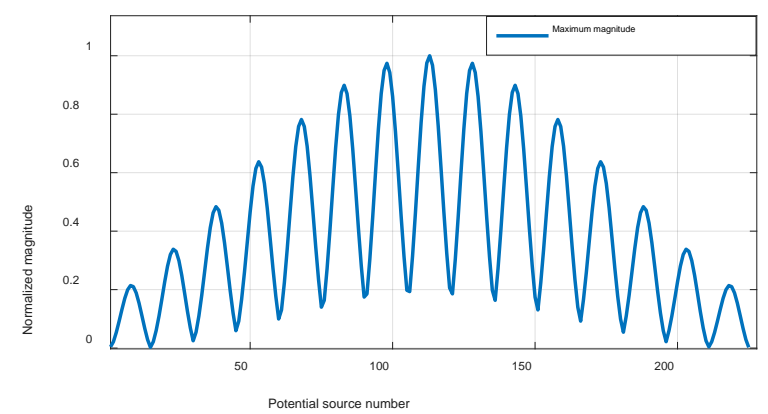

b)

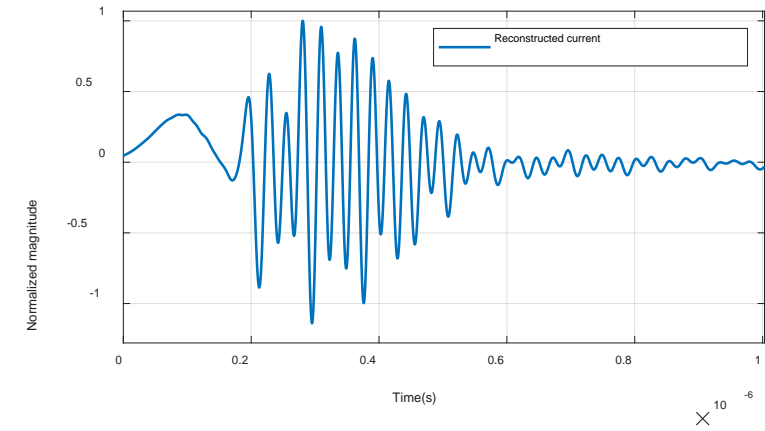

Fig. 9. (a) Maximum Focusing magnitudes, (b) Reconstructed excitation form.

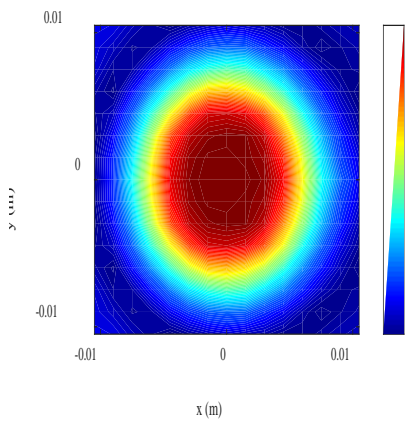

a) Measured $H_{z}$

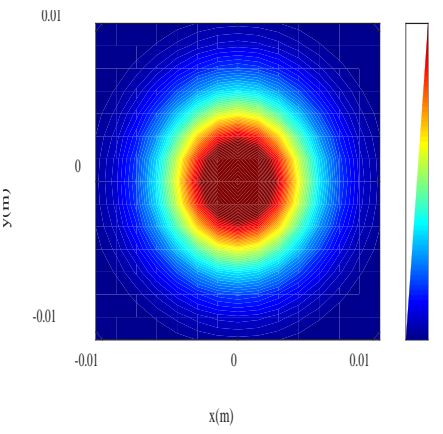

b) Reconstructed $H_{z}$
Fig. 10. (a) Radiated $H_{z}$ field cartography at $t_{0}=10.1 \mu \mathrm{s}$, (b) Recnstructed $H_{z}$ field cartography at $t_{0}=10.3 \mu \mathrm{s}$.

a)

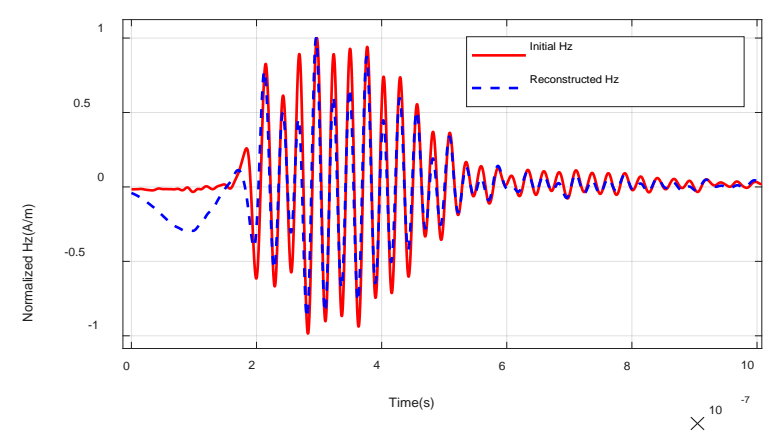


b)

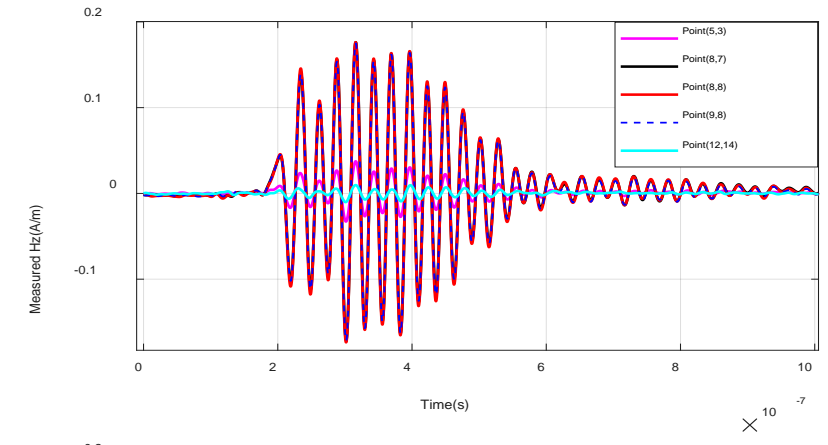

c)

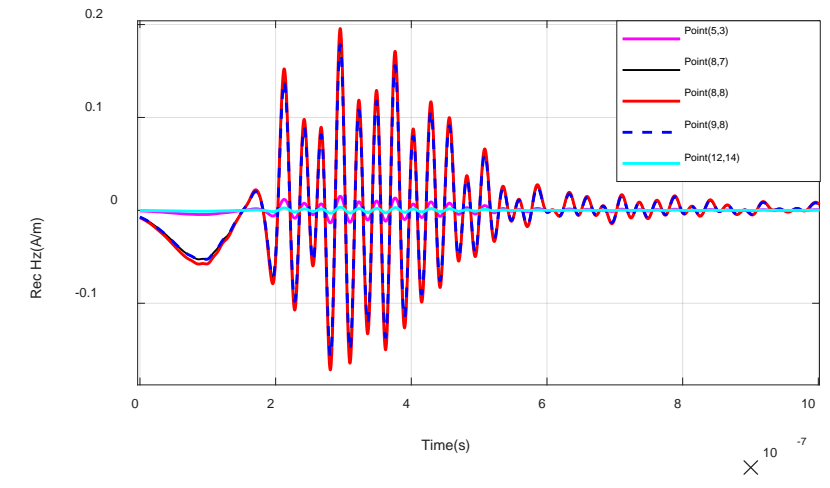

Fig. 11. (a) Comparaison between initial and reconstructed $H_{z}$ at measurement point $(0,0,0)$ at turn ON, (b) TD reconstructed fields at Turn ON, (c) TD measured magnetic fields at Turn ON.

\section{CONCLUSION}

This paper presents a Time domain Electromagnetic inverse method based on Time Reversal technique, in the near field region. The aim of the proposed methodology is to describe emissive source position and determining its corresponding excitation signal. The interest behind this work is to present a full Time Domain study of the radiating element reconstruction. In addition, the proposed approach is a simple and a low cost procedure. Indeed, not long ago, the TD EM inverse method becomes an effective alternative for modelling non-sinusoidal perturbations. Simulations have shown the potential of the TD analysis to identify EM field distributions. Its definitions are advantageous over the FD techniques, which needs to repeat the whole process at each frequency, especially in the transient regime analysis. This will save a valuable calculation time. To validate our approach, experimental measurements on basis of a simple structure were carried out. According to the obtained results, the proposed method shows a good agreement between the reconstructed magnetic field cartography and the measured distribution, and it succeeds in identifying the equivalent dipole parameters and determining the current waveform.

\section{REFERENCES}

[1] L. Beghou, F. Costa and L. Pichon, "Detection of Electromagnetic Radiations Sources at the Switching Time Scale Using an Inverse Problem-Based Resolution Method-Application to Power Electronic Circuits," in IEEE Transactions on Electromagnetic Compatibility, vol. 57, no. 1, pp. 52-60, Feb. 2015.

[2] J. Ben Hadj Slama and S. Saidi, "Coupling the electromagnetic inverse problem based on genetic algorithms with Moment's method for EMC of circuits," Melecon 2010 - 2010 15th IEEE Mediterranean Electrotechnical Conference, Valletta, 2010, pp. 709-714.

[3] S. Saidi and J. Ben Hadj Slama, "The PZMI and Artificel Neural Network to identify the electromagnetic radiation sources," 2012 16th IEEE
Mediterranean Electrotechnical Conference, Yasmine Hammamet, 2012, pp. 412-416.

[4] S. Saidi and J. Ben Hadj Slama, "A Near-Field Technique Based on PZMI, GA, and ANN: Application to Power Electronics Systems," in IEEE Transactions on Electromagnetic Compatibility, vol. 56, no. 4, pp. 784791, Aug. 2014

[5] F. Benyoubi, L. Pichon, M. Bensetti, Y. Le Bihan and M. Feliachi, "An Efficient Method for Modeling the Magnetic Field Emissions of Power Electronic Equipment From Magnetic Near Field Measurements," in IEEE Transactions on Electromagnetic Compatibility, vol. 59, no. 2, pp. 609-617, April 2017.

[6] P. F. Lopez, A. Ramanujan, Y. V. Gilabert, C. Arcambal, A. Louis and B. Mazari, "A Radiated Emission Model Compatible to a Commercial Electromagnetic Simulation Tool," 2009 20th International Zurich Symposium on Electromagnetic Compatibility, Zurich, 2009, pp. 369-372.

[7] I. Briki, L. Pichon and J. Ben Hadj Slama, "Shielding Effectiveness of Perforated Screens Through an Inverse Problem-Based Resolution," in IEEE Transactions on Magnetics, vol. 52, no. 3, pp. 1-4, March 2016.

[8] B. Zitouna and J. Ben Hadj Slama, "Development and validation of analytic equations of the electromagnetic fields radiated by the elementary dipoles in time domain," 10th International Multi-Conferences on Systems, Signals \& Devices 2013 (SSD13), Hammamet, 2013, pp. 1-6.

[9] B. Zitouna and J. Ben Hadj Slama, "Enhancement of Time-Domain Electromagnetic Inverse Method for Modeling Circuits Radiations," in IEEE Transactions on Electromagnetic Compatibility, vol. 58, no. 2, pp. 534-542, April 2016.

[10] B. Zitouna and J. Ben Hadj Slama, "Time domain electromagnetic inverse method for non-sinusoidal circuits," 2015 World Symposium on Mechatronics Engineering \& Applied Physics (WSMEAP), Sousse, 2015, pp. 1-6.

[11] Y. Kuznetsov et al., "Localization of the equivalent sources on the PCB surface by using ultra-wideband time domain near-field measurements," 2016 International Symposium on Electromagnetic Compatibility - EMC EUROPE, Wroclaw, 2016, pp. 1-6.

[12] Fink, M., Cassereau, D., Derode, A., Prada, C., Roux, P., Tanter, M., ... \& Wu, F. (2000). Time-reversed acoustics. Reports on progress in Physics, 63(12), 1933.

[13] F. Rachidi, M. Rubinstein and M. Paolone, Electromagnetic Time Reversal: Application to Electromagnetic Compatibility and Power Systems, Wiley, 2017

[14] M. Benhamouche, L. Bernard, L. Pichon and D. Lesselier, "ThreeDimensional Generalized Finite-Difference Modeling of Electromagnetic Time Reversal: Impact of the Density of Dipoles for the Localization of a Dielectric Obstacle in Free Space," in IEEE Transactions on Magnetics, vol. 48, no. 2, pp. 359-362, Feb. 2012.

[15] I. El Baba, S. Lallechere and P. Bonnet, "Electromagnetic Time-Reversal for reverberation chamber applications using FDTD," 2009 International Conference on Advances in Computational Tools for Engineering Applications, Zouk Mosbeh, 2009, pp. 157-162.

[16] R. Razzaghi, G. Lugrin, M. Paolone, F. Rachidi, Electromagnetic Time Reversal Applied to Fault Location in Power Networks, Chapter 7 of " Electromagnetic Time Reversal: Application to Electromagnetic Compatibility and Power Systems", pp. 217-274, Wiley, 2017

[17] M. Kafal, A. Cozza and L. Pichon, "Locating Multiple Soft Faults in Wire Networks Using an Alternative DORT Implementation," in IEEE Transactions on Instrumentation and Measurement, vol. 65, no. 2, pp. 399-406, Feb. 2016.

[18] P. Kosmas and C. M. Rappaport, "Time reversal with the FDTD method for microwave breast cancer detection," in IEEE Transactions on Microwave Theory and Techniques, vol. 53, no. 7, pp. 2317-2323, July 2005.

[19] T. K. Sarkar and M. S. Palma, "Electromagnetic Time Reversal: What does it imply?," 2016 URSI International Symposium on Electromagnetic Theory (EMTS), Espoo, 2016, pp. 30-33. 